\title{
Characteristics of Magnetic Clouds and Interplanetary Coronal Mass Ejections which Cause Intense Geomagnetic Storms
}

\author{
Chin-Chun $\mathrm{Wu}^{1, *}$, Natchimuthuk Gopalswamy ${ }^{2}$, Ronld Paul Lepping ${ }^{3}$, and Seiji Yashiro ${ }^{2,4}$ \\ ${ }^{1}$ Naval Research Laboratory, Washington, DC, USA \\ ${ }^{2}$ Solar Physics Laboratory, Solar System Exploration Division, NASA/GSFC, Greenbelt, Maryland, USA \\ ${ }^{3}$ Heliosphysics Science Division, NASA/GSFC, Greenbelt, Maryland, USA \\ ${ }^{4}$ The Physics Department, The Catholic University of America, Washington, DC, USA
}

Received 18 March 2012, accepted 26 September 2012

\begin{abstract}
We present the results of a statistical data analysis of the geo-effectiveness of non-magnetic-cloud interplanetary coronal mass ejections (ICMEs) and compare them with those of magnetic-cloud (MC) interplanetary coronal mass ejections observed during solar cycle 23. (The term ICME as used here will refer to a non-MC ICME.) The starting point of this investigation is the set of intense geomagnetic storms $\left(D s t_{\min } \leq-100 \mathrm{nT}\right.$ ) of solar cycle 23 between 1996 and 2005 . We also compare the solar source locations of the ICMEs with those of the MCs. The source locations of the solar disturbances are, on average, closer to the Sun-Earth line for the MCs than for the ICMEs. There is an anomaly for the location of the related solar sources: no event came from the region between the solar equator plane and $10^{\circ} \mathrm{S}$ (south) of that plane. The primary results are listed as follows. The average duration of these MCs is slightly longer ( $7 \%)$ than that of ICMEs. The average geomagnetic storm intensity for the MCs is higher than that for the ICMEs and CIRs formed by high-speed streams from coronal holes, especially for the events associated with $\mathrm{X}$ class flares. The relevant average magnetic field component, i.e., $\left|B z_{\min }\right|$, is more intense within the MCs than within the ICMEs. The average solar wind speed is similar for both MCs and ICMEs. Maximum solar wind speed is higher within ICMEs than within MCs. Maximum solar wind proton density is higher for MCs than for ICMEs.
\end{abstract}

Key words: Magnetic cloud, Interplanetary coronal mass ejection, Geomagnetic storm, Solar flare, Corotating interaction region Citation: Wu, C. C., N. Gopalswamy, R. P. Lepping, and S. Yashiro, 2013: Characteristics of magnetic clouds and interplanetary coronal mass ejections which cause intense geomagnetic storms. Terr. Atmos. Ocean. Sci., 24, 233-241, doi: 10.3319/TAO 2012.09.26.03(SEC)

\section{INTRODUCTION}

A magnetic cloud (MC) is defined as a region of a high strength magnetic-field, low proton temperature, low proton $\beta$, and smoothly-changing (rotating) magnetic field (Burlaga et al. 1981). Magnetic clouds are often preceded by upstream sheaths in which the plasma is usually hot and dense and the magnetic field is extremely turbulent (e.g., Tsurutani and Gonzalez 1997, and reference therein). The front "boundary" of the sheath may be a shock, a shock-like structure, a pressure pulse or a sharp rise in density, temperature, or velocity. About 1/4 of observed MCs have no upstream pressure pulse/shock, but all have a density increase (e.g., Wu and Lepping 2002). It is now generally believed that MCs are an important subset of ICMEs (interplanetary

\footnotetext{
* Corresponding author

E-mail: chin-chun.wu@nrl.navy.mil
}

coronal mass ejections) or are contained within them, since $\sim 90 \%$ of MCs drove geomagnetic storms with $D s t_{\min }$ (minimum $D s t$ observed during a geomagnetic storm) $\leq-30 \mathrm{nT}$ (e.g., Wu et al. 2003, 2006; Wu and Lepping 2007, 2008). Conversely, Wu and Lepping (2011) recently reported that six MCs are not associated with ICMEs by investigating 91 MCs and 307 ICMEs occurred in the period between 1996 and 2006. The average occurrence rate of MCs is $\sim 9.5 \mathrm{MCs} \mathrm{yr}^{-1}$ for the period 1995 - 2003 (e.g., Lepping et al. 2006; Wu et al. 2006; Wu and Lepping 2008) but dropped slightly to $\sim 8.6$ for the period $1995-2006$ (Wu and Lepping 2011). The average occurrence rate is higher for ICMEs ( 29.6/year) than for MCs for the period of $1996-2005$ (e.g., Richardson and Cane 2010), within which $78 \%$ or 231 out of 296 ICMEs drove geomagnetic storms with $D s t_{\min }$ $\leq-30 \mathrm{nT}$. Gopalswamy (2006) compared the properties of 
MCs and non-cloud ICMEs in general. Here we attempt to compare MCs-associated with non-MC-associated ICMEs for cases that cause strong geomagnetic storms. For simplicity, the term ICME as used henceforth will refer to a nonMC-associated ICME; and the term MC as used henceforth will refer to a MC-associated ICME.

Studying the interplanetary causes of intense geomagnetic storms (Dst < -100 nT) during solar cycle 23 (1997 - 2005), Gonzalez et al. (2007) found that the most common interplanetary structures leading to the development of an intense storm were: magnetic clouds, sheath fields, sheath fields followed by a magnetic cloud and co-rotating interaction regions leading high speed streams. Zhang et al. (2007) studied 88 major (or intense) geomagnetic storms with $D s t_{\min } \leq-100 \mathrm{nT}$ over the period of $1996-2005$ caused by MCs, ICMEs and co-rotating interaction regions (CIRs). They found (1) $60 \%, 27 \%$, and $13 \%$ of the major geomagnetic storms were caused by a single coronal mass ejection (CME)/ICME, multiple CMEs/ICMEs, and coronal holes or CIRs, respectively; (2) 63\%, 12\%, $13 \%$, and $12 \%$ of major geomagnetic storms were related to solar active regions, quiet-Sun regions, coronal holes, and no signature areas, respectively; and (3) the locations of solar source regions were cataloged according to active region and quiet Sun. More than $87 \%[60 \%+27 \%$ in above item (1)] of major geomagnetic storms are caused by either MCs or ICMEs. This motivates us to investigate if there are any distinguishing properties of ICMEs and MCs that caused the major geomagnetic storms. In this study, we separate the causes of major geomagnetic storms into three groups: MC-related, ICME-related, and CIR-related. There are three kinds of geomagnetic storms which are cataloged by the driving sources: (1) "sheath storm" wherein a geomagnetic storm is primarily driven by the southward IMF in the sheath region, and $D s t_{\text {min }}$ occurred within sheath region; (2) "ejecta storm" such that a geomagnetic storm is primarily driven by the southward IMF in the ejecta (MC or ICME) region, and $D s t_{\text {min }}$ occurred within ejecta region; and (3) "two-step storm" demonstrating that a geomagnetic storm is primarily driven by the southward IMF in both the sheath and ejecta regions, and $D s t_{\min }$ occurred within the ejecta region. If the magnetic fields are southward both in regard to the sheath and solar ejecta, a two-step main phase storm can result (e.g., Gonzalez et al. 2001). A two-step storm is defined as a storm in which a Dst decrease (induced by the southward fields in the sheath) does not fully recover to the pre-storm level before a second Dst decrease (induced by the southward fields in the solar ejecta) follows (e.g., Kamide et al. 1998) We will investigate the differences between these three kinds of storms.

\section{DATA AND ANALYSIS}

Four data sets are used in this study. (1) 88 intense geomagnetic storms (with $D s t_{\min } \leq-100 \mathrm{nT}$ which occurred from 1996 to 2005) as taken from Zhang et al. (2007). Information on Solar source locations, interplanetary shocks, interplanetary structures, e.g., MCs, ICMEs, or CIRs are included. A storm may be associated with a single MC/ICME, a complex solar wind flow produced by multiple interacting ICMEs (e.g., Yermolaev and Yermolaev 2008), the sheath itself, or the combination of the sheath with ICMEs/MCs wherein the sheath is the region between an interplanetary shock and an ICME. (2) The MCs are adopted from Lepping et al. (2006), and are listed in http://lepmfi.gsfc.nasa. gov/mfi/mag cloud pub1.html, and from Huttunen et al. (2005). (3) The ICMEs are adopted from Richardson and Cane (2010). If an ICME was not identified as an MC (or not covering the near region of a MC within a few hours), we list it as a non-MC ICME. Finally, (4) solar wind plasma and magnetic field data (from the WIND or ACE spacecraft) are provided by the NASA Space Physics Data Facility (http://vho.nasa.gov/mission/wind/swe gsfc/ and http:// vho.nasa.gov/mission/wind/mfi/).

\subsection{The Association Between CMEs and ICMEs}

It is a complex task to determine the association between ICME and CME (e.g., Eselevich et al. 2009). In this study, we picked 88 events which were identified by Zhang et al. (2007). They used an interactive process with multiple steps which contains three major steps: (1) find all candidate front-side halo CMEs within a 120-hour-long search window before the arrival time of the ICME-driven shock; (2) reduce the search window by estimating the CME transit time based on in situ solar wind velocities at the location of shock arrival; and (3) for each remaining candidate CME in the search window, consider whether the CME speed at the Sun is consistent with the $1 \mathrm{AU}$ transit speed implied by an association with the $1 \mathrm{AU}$ shock/ICME and with the in situ solar wind speed [More detail information can be found in previous study by Zhang et al. (2007)].

\subsection{Identification of Solar Source Locations}

It is usually straightforward to identify the solar source of an ICME, but the results are sometimes ambiguous. For example, Wang et al. (2011) found that 231 CMEs source locations cannot be identified due to poor data, and 325 CMEs have no evident eruptive signatures in the field of view of EIT as a result of investigating 1078 Large Angle and Spectrometric Coronagraph (LASCO) CMEs listed in Coordinated Data Analysis Workshop (CDAW) catalog during the interval of 1977 - 1998.

The source locations for the studied events are adapted from a previous study (Zhang et al. 2007). First, they find a front side halo (full or partial) CME at a reasonable earlier time which depends on the transit time of the CME from the 
Sun to the Earth (e.g., Webb et al. 2000; Zhang et al. 2003). Then, they use a cause-and-effect relationship between solar wind IP events to help justify the finding. In this study 88 ICMEs have been shown to cause severe geomagnetic storms when they passed the Earth. The relationship between CMEs and ICMEs is described in the above section.

There are three ways to identify the locations of solar sources (ejecta) according to Zhang et al. (2007). They are by: (1) using observations from the SOHO spacecraft wherein CMEs near the Sun are observed by the LASCO $\mathrm{C} 2$ and $\mathrm{C} 3$ coronagraphs which have fields of view of 2 - 6 solar radii $\left(R_{s}\right)$ and 4 - $30 R_{s}$, respectively; (2) identifying the surface features of CMEs in the source region with observations from the SOHO Extreme-Ultraviolet Imaging Telescope (EIT) which takes images of the Sun's corona over the full disk up to $1.5 R_{s}$ altitude; and (3) using traditional synoptic data, daily NOAA solar event reports which include data on soft X-ray flares, filament eruptions, and active regions.

\section{RESULTS}

Figure 1 shows the solar source locations of $43 \mathrm{MCs}$ and 32 ICMEs causing intense geomagnetic storms. CIRs caused 13 intense storms, which are not shown in Fig. 1. Most events occurred within a $30^{\circ}$ latitude of the solar equator.

For solar sources located in the northern hemisphere, the average latitudes are $8^{\circ} \mathrm{N}$ for MCs and $14^{\circ} \mathrm{N}$ for ICMEs, and the average longitudes are $4^{\circ} \mathrm{W}$ for MCs and $12^{\circ} \mathrm{W}$ for ICMEs. For solar sources located in the southern hemisphere, the average latitudes are $21^{\circ} \mathrm{S}(\mathrm{MCs})$ and $14^{\circ} \mathrm{S}$ (ICMEs), and the average longitudes are $15^{\circ} \mathrm{W}$ (MCs) and $10^{\circ} \mathrm{W}$ (ICMEs). The average latitude of source locations for ICMEs $\left(\sim 14^{\circ}\right)$ is higher than for MCs $\left(\sim 11^{\circ}\right)$. The average source longitude of ICMEs is $10^{\circ} \mathrm{W}$, compared to $7^{\circ} \mathrm{W}$ for MCs (see also Table 1). The $\sigma$ 's ( $\sigma$ : standard deviation) for MCs are $2.87^{\circ}$ for latitude and $5.03^{\circ}$ for longitude, and the $\sigma$ 's for ICMEs are $2.48^{\circ}$ for latitude and $3.08^{\circ}$ for latitude. The larger $\sigma$ of latitude and longitude for MCs are caused by two abnormal events which are located at S58W05 and S20W85.

Figure 2 shows Dst distributions of storms caused separately by MCs, ICMEs, and CIRs. The averages of $D s t_{\min }$ $\left(<D s t_{\min }>\right)$ are $-175,-161$ and $-129 \mathrm{nT}$ for the events caused by the MCs, ICMEs, and CIRs, respectively. The spread in values, measured by $\sigma$, are 13.6, 11.6, and $14.4 \mathrm{nT}$ for the MCs, ICMEs, and CIRs, respectively. The median $D s t_{\min }$ values are $-142,-147$, and $-116 \mathrm{nT}$ for MCs, ICMEs, and CIRs, respectively. Table 2 also summarizes these values. It is clear that, on the average, the $\mathrm{MC}$ storms are a bit stronger $(\sim 9 \%)$ than the ICME storms. Figure 3 shows distributions of solar wind properties of ICMEs and MCs (see Table 3). Most solar wind properties are similar for MCs and ICMEs, except for their magnetic fields. The average speeds of MCs and ICMEs are very similar (less than $1 \%$ difference), but the median speed of ICMEs is $\sim 14 \%$ higher.

Table 4 summarizes the flare association of $\mathrm{MC}$ and ICME events. There are 27 ICMEs and 28 MCs that have associated flares, and the $\left\langle D s t_{\min }\right\rangle$ driven by those MCs $(-198 \mathrm{nT})$ is $\sim 18 \%$ higher than that associated with the ICMEs $(-168 \mathrm{nT})$. The $\sigma$ 's are $100 \mathrm{nT}$ for MCs and $67 \mathrm{nT}$ for ICMEs. In addition, the $\left\langle D s t_{\min }>\right.$ driven by flare-associated MCs (-198 nT) is $\sim 13 \%$ higher than that for all MCs $(-175 \mathrm{nT})$. For the 27 ICMEs related with flares, one event was related to a B class flare; 9 events were related to a $\mathrm{C}$ class flare; 6 events were related to an M class flare; and 12 events were related to an $\mathrm{X}$ class flare. For the 28 flare related MCs events, nine events were related to a $\mathrm{C}$ class flare; 8 events were related to an $\mathrm{M}$ class flare; and, 12 events were related to an X class flare. Forty one percent (41\%) of ICMEs and $29 \%$ of MCs, related to intense storms, were associated with $\mathrm{X}$ class flares. This explains why the average speed $\left(V_{\max }\right)$ of the ICMEs was higher than that for the MCs because CME speeds are correlated with X-ray flare size (Yashiro et al. 2005).

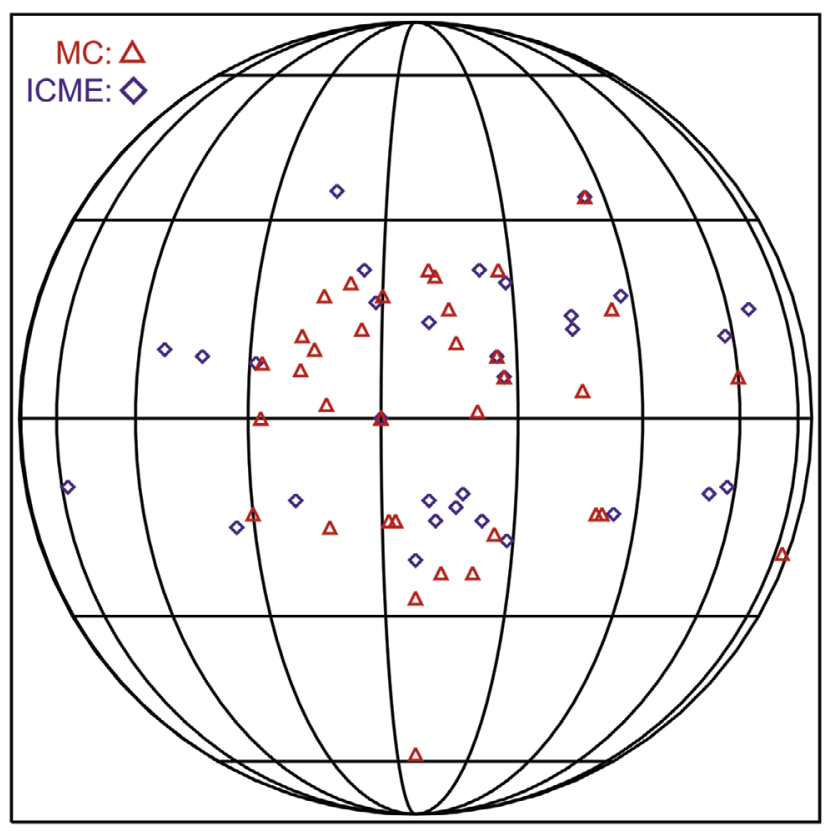

Fig. 1. Solar source locations for MCs (triangles) and non-magneticcloud ICMEs (diamonds) the grid spacing is $20^{\circ}$ in latitude and longitude.

Table 1. Averages of solar source locations for MCs and ICMEs.

\begin{tabular}{l|cccc}
\hline Source location & $\mathrm{MC}$ & $\sigma^{\mathrm{a}}$ & ICMEs & $\sigma^{\mathrm{a}}$ \\
Latitude & $11^{\circ}$ & $2.87^{\circ}$ & $14^{\circ}$ & $2.48^{\circ}$ \\
Longitude & $7^{\circ} \mathrm{W}$ & $5.03^{\circ}$ & $10^{\circ} \mathrm{W}$ & $3.08^{\circ}$ \\
\hline
\end{tabular}

a: $\sigma$ - standard deviation. 


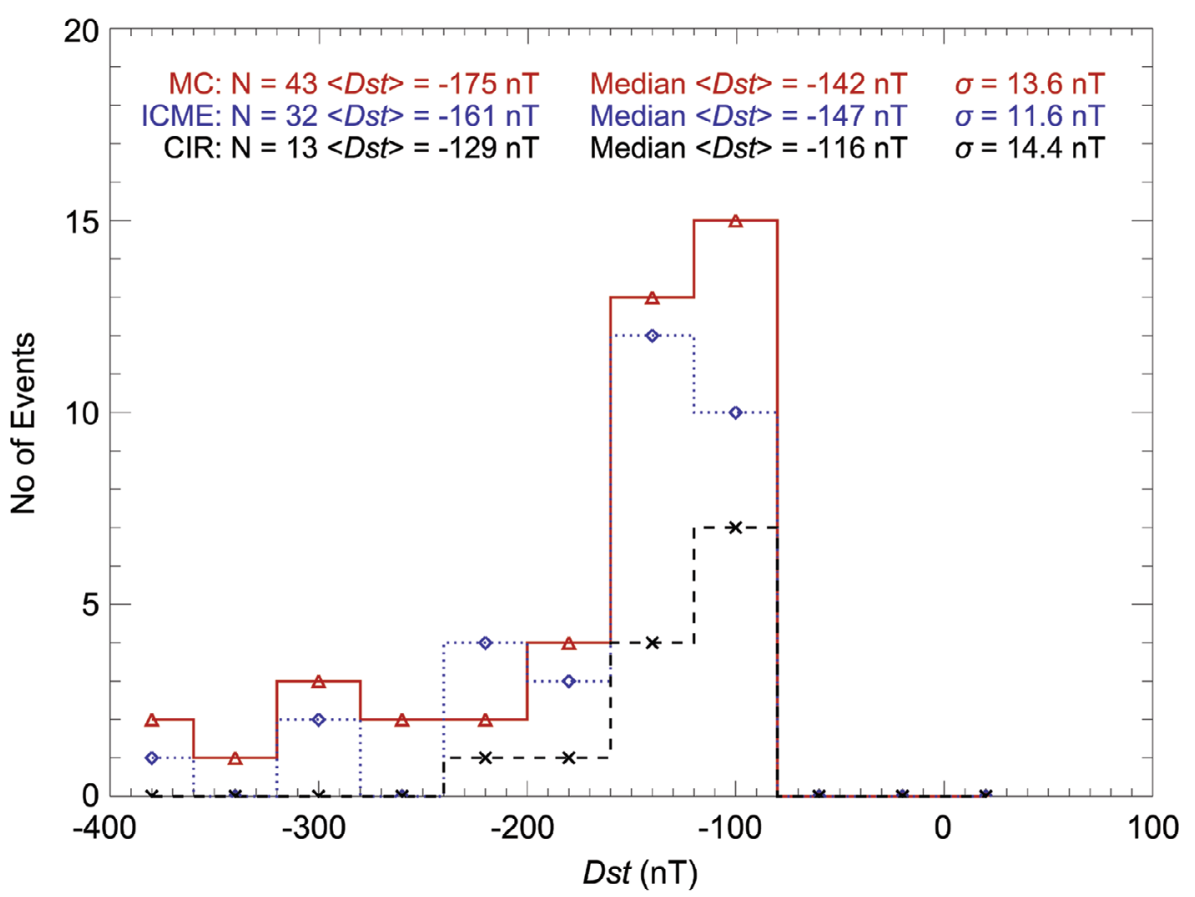

Fig. 2. Histograms of storm Intensity ( $D s t_{\min }$ ) for MCs (triangles), ICMEs (diamonds), and CIRs (crosses) for the period $1996-2005$ in which, $N p$ $(\max )$ and $V(\max )$ are the peak proton density and velocity within MCs, ICMEs, or CIRs.

Table 2. Averages of Intensity for intense geomagnetic storms caused by MCs, ICMEs, and CIRs during the interval of $1996-2005$.

\begin{tabular}{l|ccc}
\hline & MCs (43) & ICMEs (32) & CIRs (13) \\
\hline$<D s t_{\min }>(\mathbf{n T})$ & -175 & -161 & -129 \\
${\text { Median } D s t_{\min }(\mathbf{n T})}^{\boldsymbol{\sigma}^{\mathbf{a}}(\mathbf{n T})}$ & -142 & -147 & -116 \\
\hline
\end{tabular}

a: $\sigma$ - standard deviation.

\section{DISCUSSION}

About $85 \%$ of major (or intense) geomagnetic storms are related to either MCs or ICMEs (e.g., Zhang et al. 2007). The other significant cases of such storms are CIRs. Among these three sources of intense geomagnetic storms, MCs are the most important: (1) about $\sim 50 \%$ of intense geomagnetic storms are caused by MCs; and, (2) the intensity of MC storms is greater than that for ICME storms.

The average magnetic field at Earth's orbit of the storm-producing MCs (14 nT) is 27\% larger than that for ICMEs (11 nT). Average speed is almost the same (less than $1 \%$ difference) for both types: $498 \mathrm{~km} \mathrm{~s}^{-1}$ (MCs) and $500 \mathrm{~km} \mathrm{~s}^{-1}$ (ICMEs). This result consists with long-term statistical study for the general population by Yermolaev et al. (2010) who investigated more than 20 years worth of solar wind data (1976 - 2000). However, the median speed of ICMEs $\left(535 \mathrm{~km} \mathrm{~s}^{-1}\right)$ is faster than that for MCs $\left(469 \mathrm{~km} \mathrm{~s}^{-1}\right)$. This speed relation is consistent with projection effects.
Storm-effective MC sources are much closer to the disk center, so the associated CMEs have intense projection effects compared to those originating at larger central meridian distances (see Gopalswamy et al. 2007). Specifically, the median speed for ICMEs is $14 \%$ higher than that for MCs. Since the average source location for ICMEs is further away from the Sun-Earth line than that for MCs (e.g., see Fig. 1 and Table 1), more energy for ICMEs to propagate to the Earth is required. For intense storms which are related to solar flares, there is a greater percentage of ICMEs (41\%) than MCs (29\%) related to X class flares, but the intensity of geomagnetic storms for MCs $\left(<D s t_{\min }>\sim-264 \mathrm{nT}\right)$ is higher than that for ICMEs $\left(<D s t_{\min }>\sim-181 \mathrm{nT}\right)$. Therefore, the average speed for ICMEs is higher than that for MCs because the energy input from the solar source for ICMEs is stronger than that for MCs. The average $B z_{\min }$ is stronger within MCs $(-21 \mathrm{nT})$ than within ICMEs $(-17 \mathrm{nT})$ which also supports the above features. The average duration is longer for MCassociated events (1.34 days) than for ICME-associated events (1.12 days). It is interesting to note that the average magnetic field strength of our MCs is $\sim 30 \%$ higher than that for the general population (e.g., Gopalswamy 2006; Yermolaev et al. 2010), stressing the importance of field strength in causing storms. Again, the stronger magnetic fields in MCs than in ICMEs also show the importance of solar source locations for MCs.

Both Fig. 1 and Table 1 show clearly: (1) most solar sources occurred within $30^{\circ}$ of the solar equator, with one exception, which is the MC-associated event that occurred 

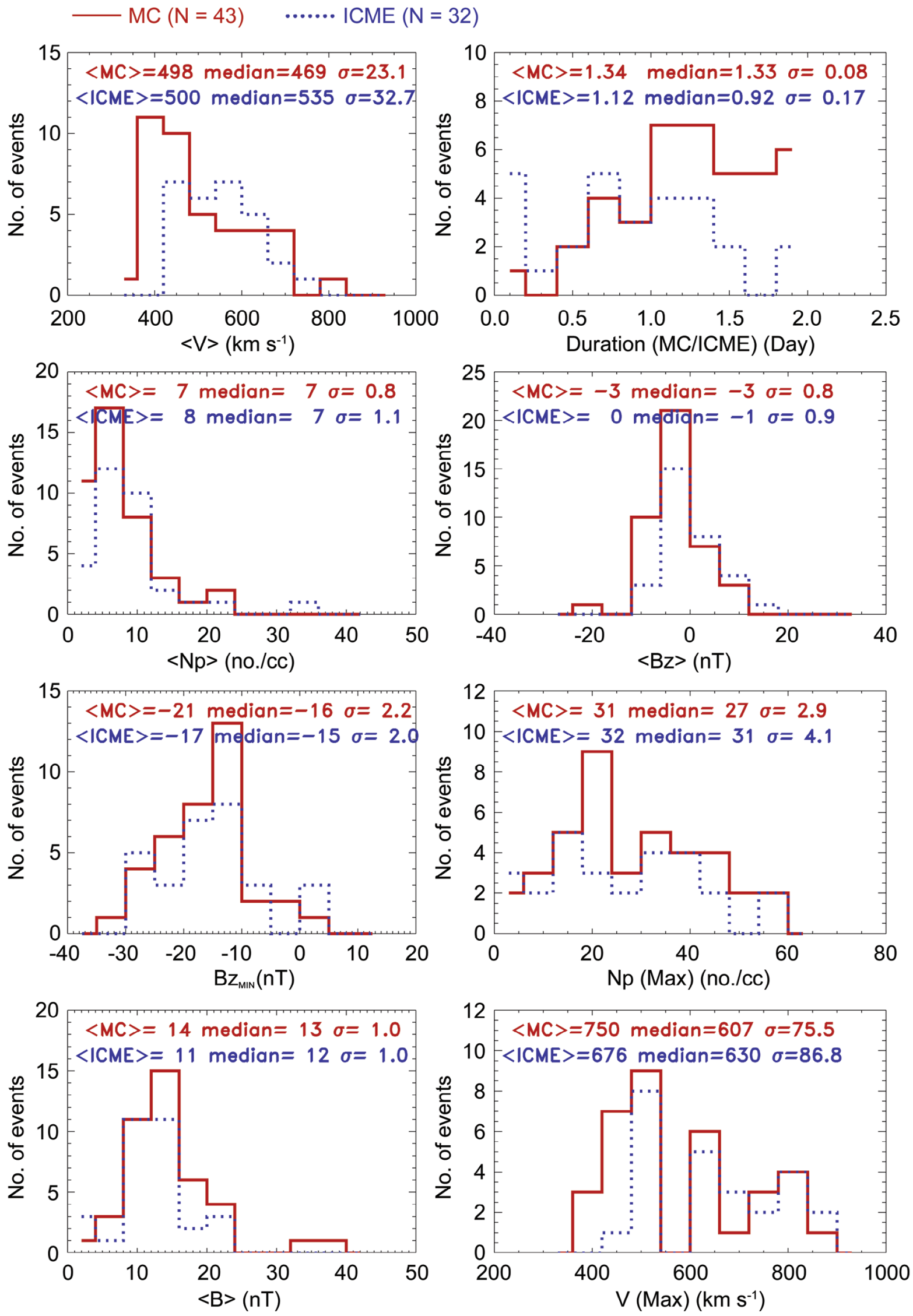

Fig. 3. Histograms of solar wind properties for storm-related MCs and ICMEs. 
Table 3. Solar wind properties of MCs and ICMEs which caused intense geomagnetic storms during the interval of 1995 - 2005.

\begin{tabular}{l|cccccc}
\hline & MCs & ICMEs & MCs & ICMEs & MCs & ICMEs \\
\cline { 2 - 7 } & Average & Average & Median & Median & $\boldsymbol{\sigma}^{\mathbf{a}}$ & $\boldsymbol{\sigma}^{\mathbf{a}}$ \\
\hline Duration (Days) & 1.34 & 1.12 & 1.33 & 0.92 & 0.08 & 0.17 \\
$\mathbf{B}(\mathbf{n T})$ & 14 & 11 & 13 & 12 & 1.0 & 1.0 \\
$\boldsymbol{B} \boldsymbol{z}_{\min }{ }^{\mathbf{b}}(\mathbf{n T})$ & -21 & -17 & -16 & -15 & 2.2 & 2.0 \\
$\boldsymbol{N} \boldsymbol{p}^{\mathbf{c}}\left(\mathbf{c m}^{-3}\right)$ & 7 & 8 & 7 & 7 & 0.8 & 1.1 \\
$\boldsymbol{N} \boldsymbol{p}_{\max }{ }^{\mathbf{d}}\left(\mathbf{c m}^{-3}\right)$ & 31 & 32 & 27 & 31 & 2.9 & 4.1 \\
$\boldsymbol{V}^{\mathbf{e}}\left(\mathbf{k m ~ s}^{-1}\right)$ & 498 & 500 & 469 & 535 & 23.1 & 32.7 \\
$\boldsymbol{V}_{\max }{ }^{\mathbf{f}}\left(\mathbf{k m ~ s}^{-1}\right)$ & 750 & 676 & 607 & 630 & 75.5 & 86.8 \\
$\left\langle\boldsymbol{B} z>^{\mathbf{g}}(\mathbf{n T})\right.$ & -3 & 0 & -3 & -1 & 0.8 & 0.9 \\
\hline
\end{tabular}

Notes: $a$ : $\sigma$ - standard deviation (nT); $b$ : Minimum Bz within a MC/ICME; $c$ : average Np within a MC/ICME; $d$ : maximum Np within a MC/ICME; $e$ : average V within a MC/ICME; f: maximum V within a MC/ICME; and $g$ : average $B z$ within a $M C / I C M E$.

Table 4. Flare association.

\begin{tabular}{|c|c|c|c|}
\hline & MCs & ICMES & CIRs \\
\hline Storms & $49 \%$ & $36 \%$ & $15 \%$ \\
\hline$<D s t_{\min }>(\mathbf{n T})$ & -175 & -161 & -129 \\
\hline Storms associated with Flares & 28 & 27 & \\
\hline$<D s t_{\min }>(\mathbf{n T})$ & -198 & -168 & \\
\hline$\sigma(\mathbf{n T})$ & 100 & 67 & \\
\hline X Class (no. of events) & 8 & 11 & \\
\hline$<D s t_{\min }>(\mathbf{n T})$ & -264 & -181 & \\
\hline$\sigma(\mathbf{n T})$ & 100 & 86 & \\
\hline M Class (no. of events) & 11 & 6 & \\
\hline$<D s t_{\min }>(\mathbf{n T})$ & -206 & -164 & \\
\hline$\sigma(\mathbf{n T})$ & 104 & 55 & \\
\hline C Class (no. of events) & 9 & 9 & \\
\hline$<D s t_{\min }>(\mathbf{n T})$ & -129 & -159 & \\
\hline$\sigma(\mathbf{n T})$ & 42 & 54 & \\
\hline B Class (no. of events) & 0 & 1 & \\
\hline$<D s t_{\min }>(\mathrm{nT})$ & & -138 & \\
\hline
\end{tabular}

at $58^{\circ} \mathrm{S}$; and (2) more sources were in the north than in the south which probably reflects the asymmetry in the locations of major active regions during solar cycle 23 (e.g., Richardson and Cane 2005; Riley et al. 2006). The solar source locations of ICMEs related to "intense storms" were on average further away from the Sun-Earth line than those of the MCs. This implies that on average the observing spacecraft were closer to the center of the solar ejecta for the MCs than for the ICMEs. The following average features show this effect. (i) The intensity of the magnetic field is stronger for MCs than for ICMEs; (ii) maximum $\left|B z_{\text {min }}\right|$ is stronger within MCs than within ICMEs; and (iii) the duration is longer for MCs than for ICMEs. The effects of items (i) and (ii) are directly connected with criteria of selection for $\mathrm{MC}$ and non-MC events. Note that the average duration of MCs is about $\sim 19 \%$ longer than that for ICMEs, but the median duration is $\sim 45 \%$ longer for MCs than for ICMEs. This implies that there are more shorter-duration ICMEs than MCs, but some ICMEs are much longer than MCs.

There is an anomaly in the locations of the related solar sources; no event came from the region between the solar equator plane and $10^{\circ} \mathrm{S}$ (south) of that plane. This feature was not observed by studying general cases over the interval 1996 - 2003 [e.g., see Fig. 9 of Gopalswamy (2006)]. The butterfly diagram of sunspot numbers might explain why this anomaly happened; there were no active regions near the solar equator. In any case, it is a mystery that the anomaly is only just south of solar equatorial plane. The solar heliospheric current sheet (HCS) might be present within this region. An interaction might have taken place between the HCS and the solar ejecta. For example, performing global three-dimensional magnetohydrodynamic numerical simulations, Wu et al. (2007b) studied this kind interaction which occurred during solar minimum for the well known event of May 12, 1997 [see Fig. 2 of Wu et al. (2007b)], and during solar maximum for solar events of October 25 - 28, 2003 [see Figs. 1 - 2 of Wu et al. (2007a)]. Figure 2 of both $\mathrm{Wu}$ et al. (2007a and b) show that the shape of the ICME was distorted, and the characteristics of ICME might have been destroyed after interacting with the HCS. This might explain why there was no solar source within this region of ejecta which drove intense geomagnetic storms. This requires further investigation in detail by performing data analysis or numerical simulation which are considered to be beyond the scope of this paper.

Gopalswamy (2006) compared the properties of 85 MCs and 109 shock-driving ICMEs that occurred during 1996 - 2003. Figure 4 of Gopalswamy (2006) shows the following differences between MCs and shock-driving IC- 
MEs: (1) magnetic field, solar wind speed, and solar wind proton density within MCs are higher than within shockdriving ICMEs; (2) thermal speed is lower within MCs than within shock-driving ICMEs; and (3) the average duration for shock-driving ICME events is longer than that for MCs. This study investigated major (intense) geomagnetic storms which occurred from 1996 to 2005, slightly longer than the period studied by Gopalswamy (2006). The following features are found in this study: (i) the average magnetic field strength for the events associated with MCs is higher than that associated with ICMEs which is consistent with the findings of Gopalswamy (2006). (ii) the average duration is higher for MCs $(\sim 32.2 \mathrm{hr})$ than for ICMEs $(\sim 26.9 \mathrm{hr})$. The results of Gopalswamy (2006) show that the average duration of shock-driving ICMEs $(\sim 38.8 \mathrm{hr})$ is much longer than that of MCs $(\sim 20.9 \mathrm{hr})$. The currently studied events are associated with major geomagnetic storms which might require a long duration southward interplanetary magnetic field to drive down the value of Dst (e.g., Wu et al. 2006). This is consistent with the average duration of the MCs for the major (intense) geomagnetic storms being much longer than the average duration of the MCs that occurred during the interval of $1996-2003$.

Characteristics of magnetic clouds (MCs), magnetic cloud-like structures (MCLs), and interplanetary coronal mass ejections (ICMEs) have been studied and compared, previously. For example, using WIND in-situ solar wind plasma and magnetic field data with a one-minute resolution, Wu and Lepping (2007) compared the characteristics of $\mathrm{MC}$ and a magnetic cloud-like structures (MCL) for events which occurred over the period of 1995 - 2003. The average duration of the cloud passage was between 14.9 and 21.0 hours with associated $D s t_{\min }$ being -45 , and $-91 \mathrm{nT}$ for MCLs and MCs, respectively. The average plasma beta was 0.1 for both MCLs and MCs. Wu and Lepping (2008) also compared the geo-effectiveness of MCs, MCLs and interplanetary (IP) shocks for the period 1995 - 2003. The associated $D s t_{\min }$ was $-91,-45,-74.6$, and $-66 \mathrm{nT}$ for MCs, MCLs, IP shocks with MCs/MCLs and IP shocks without MCs/MCLs, respectively. Recently, using OMNI hourly data, Yermolaev et al. (2010) studied the occurrence rate and geo-effectiveness of large-scale solar wind structures for the time interval of 1976 - 2000. The average duration for MCs and ICMEs was 25 and 29 hours, respectively. The associated geomagnetic activity $\left(D s t_{\min }\right)$ was -52.1 and $-21.1 \mathrm{nT}$. The average beta value was 0.016 and 0.31 , respectively. More recently, $\mathrm{Wu}$ and Lepping (2011) performed a statistical study of MCs and ICMEs that occurred in solar cycle 23 (1996 - 2006). They found an average duration was 20.6 hours for the MCs and 29.6 hours for the ICMEs that they studied. Their associated $D s t_{\min }$ were -86.4 and $-79.7 \mathrm{nT}$, and average beta values were 0.0972 and 0.242 , respectively. All these previous studies suggest: (1) MCs are the most important storm-causing structures, (2) the duration is shorter for MCs than for ICMEs, and (3) the plasma $\beta$ is smaller for MCs than for ICMEs.

In this study, we investigate the characteristics of MCassociated ICMEs and non-MC associated ICMEs for the events followed by severe geomagnetic storms $\left(D s t_{\min }<\right.$ $-100 \mathrm{nT})$. The average of the related $D s t_{\min }$ is -175 and $-161 \mathrm{nT}$ for MC-associated and non-MC-associated ICMEs, respectively. This result is consistent with previous studies as discussed above. The average duration is 1.34 and 1.12 days (or 32.2 and 26.9 hours) for MC- and non-MC-associated ICME events, respectively. Since all the events are associated with ICMEs, the one that contains a MC (two or more MCs) is likely to be more geo-effective. The difference between MCs and ICMEs, where changing field characteristics and low $\beta_{p}$ are emphasized for the MCs, is one of the major reasons for the difference of their average characteristics (Wu and Lepping 2011).

Lepping et al. (2011) identified $18 \mathrm{MCs}$ for the period 2007 - 2009. The average duration of MCs (15.2 hours) is shorter [by 49\%, (15.2 -29.6)/29.6 × 100\% = -49\%] than that found in the previous solar cycle (1995 - 1997) which is 29.6 hours. Recently, Kilpua et al. (2012) introduced a new terminology; the "ICME-like structure" is used for ICMEs with a small flux-rope. The small flux-rope previously discussed has been described by Feng et al. (2008). Kilpua et al. (2012) found that the duration of a typical ICME-like structure is less than 10 hours [see Table 1 in Feng et al. (2008) or Kilpua et al. (2012)], and its magnetic field is weak $(<7 \mathrm{nT})$. Kilpua et al. (2012) identified a total of 85 ICMEs during the period $2007-2010$. The durations of ICMEs are $19.8,22.4$, and 25.9 hours for the periods of January 2007 - June 2008, July 2008 - June 2009, and July 2009 - December 2010, respectively. The overall duration of ICMEs is 23.9 hours $[=(19.8 \times 11+22.4 \times 38+25.9 \times 50) /(11+38+$ 50)] according to data listed in Table 4 as listed by Kilpua et al. (2012). The average duration of ICMEs (23.9 hours) is about $36 \%$ [ $=(23.9-15.2) / 23.9]$ longer than that for MCs ( 15.2 hours). This is slightly $5 \%(=36 \%-31 \%)$ larger than the typical period $[\sim 31 \%=(29.6-20.5) / 29.6]$ found for the cycle 23 during 1995 - 2006.

\section{CONCLUSIONS}

We investigated all intense geomagnetic storms which had $D s t_{\min } \leq-100 \mathrm{nT}$ during the interval of $1996-2005$. We found that magnetic clouds are the most important interplanetary structures with respect to inducing intense geomagnetic storms. The main results of this study are: (1) there are $\sim 49 \%, 36 \%$, and $15 \%$ of the intense geomagnetic storms caused by MC-associated ICMEs, non-MC-associated ICMEs, and CIRs, respectively, (2) the average duration of these MCs is slightly longer ( 19\%) than that of the ICMEs, (3) the average geomagnetic storm intensity for the MCs is higher than that of the ICMEs or CIRs, especially for events 
associated with X class flares, (4) the solar source locations are closer to the Sun-Earth line for the MCs than for the ICMEs, (5) the average magnetic field strength and $B z_{\min }$ are stronger within MCs than within ICMEs, (6) the average solar wind speed is similar for both MCs and ICMEs, and (7) both average and maximum solar wind proton density are higher for ICMEs than for MCs.

Acknowledgements We thank the Wind SWE and MFI teams and the National Space Science Data Center at Goddard Space Flight Center for Wind data management and for providing the Wind solar wind plasma and magnetic field data, and the team at Kyoto University, Kyoto, Japan for providing the Dst data. This study is supported partially by NASA's LWS program via grants NNH09AM46I (CCW and RPL), and NRL 6.1 program (CCW).

\section{REFERENCES}

Burlaga, L., E. Sittler, F. Mariani, and R. Schwenn, 1981: Magnetic loop behind an interplanetary shock: Voyager, Helios, and IMP 8 observations. J. Geophys. Res., 86, 6673-6684, doi: 10.1029/JA086iA08p06673. [Link]

Eselevich, V. G., V. M. Bogod, I. V. Chashey, M. V. Eselevich, and Y. I. Yermolaev, 2009: Comment on the paper "CAWSES November 7-8, 2004, Superstorm: Complex Solar and Interplanetary Features in the PostSolar Maximum Phase," B. T. Tsurutani, E. Echer, F. L. Guarnieri, and J. U. Kozyra. Geophys. Res. Lett., 35 (2008). Geomagn. Aeron., 49, 133-135, doi: 10.1134/ S0016793209010186. [Link]

Feng, H. Q., D. J. Wu, C. C. Lin, J. K. Chao, L. C. Lee, and L. H. Lyu, 2008: Interplanetary small- and intermediate-sized magnetic flux ropes during 1995-2005. $J$. Geophys. Res., 113, 105, doi: 10.1029/2008JA013103. [Link]

Gonzalez, W. D., A. L. C. Gonzalez, J. H. A. Sobral, A. D. Lago, and L. E. Vieira, 2001: Solar and interplanetary causes of very intense geomagnetic storms. J. Atmos. Sol.-Terr. Phys., 63, 403-412, doi: 10.1016/S1364-68 26(00)00168-1. [Link]

Gonzalez, W. D., E. Echer, A. L. Clua-Gonzalez, and B. T. Tsurutani, 2007: Interplanetary origin of intense geomagnetic storms $(D s t<-100 \mathrm{nT})$ during solar cycle 23. Geophys. Res. Lett., 34, 101, doi: 10.1029/2006GL 028879. [Link]

Gopalswamy, N., 2006: Properties of interplanetary coronal mass ejections. Space Sci.Rev., 124, 145-168, doi: 10.1007/s11214-006-9102-1. [Link]

Gopalswamy, N., S. Yashiro, and S. Akiyama, 2007: Geoeffectiveness of halo coronal mass ejections. J. Geophys. Res., 112, doi: 10.1029/2006JA012149. [Link]

Huttunen, K. E. J., R. Schwenn, V. Bothmer, and H. E. J.
Koskinen, 2005: Properties and geoeffectiveness of magnetic clouds in the rising, maximum and early declining phases of solar cycle 23. Ann. Geophys., 23, 625-641, doi: 10.5194/angeo-23-625-2005. [Link]

Kamide, Y., N. Yokoyama, W. Gonzalez, B. T. Tsurutani, I. A. Daglis, A. Brekke, and S. Masuda, 1998: Two-step development of geomagnetic storms. J. Geophys. Res., 103, 6917-6921, doi: 10.1029/97JA03337. [Link]

Kilpua, E. K. J., L. K. Jian, Y. Li, J. G. Luhmann, and C. T. Russell, 2012: Observations of ICMEs and ICME-like solar wind structures from 2007-2010 using near-earth and STEREO observations. Sol. Phys., 281, 391-409, doi: 10.1007/s11207-012-9957-0. [Link]

Lepping, R. P., D. B. Berdichevsky, C. C. Wu, Z. Szabo, T. Narock, F. Mariani, A. J. Lazarus, and A. J. Quivers, 2006: A summary of WIND magnetic clouds for years 1995-2003: Model-fitted parameters, associated errors and classification. Ann. Geophys., 24, 215-245, doi: 10.5194/angeo-24-215-2006. [Link]

Lepping, R. P., C. C. Wu, D. B. Berdichevsky, and A. Szabo, 2011: Magnetic clouds at/near the 2007-2009 solar minimum: Frequency of occurrence and some unusual properties. Sol.Phys., 274, 345-360, doi: 10.1007/s112 07-010-9646-9. [Link]

Richardson, I. G. and H. V. Cane, 2005: The 150 day quasi-periodicity in interplanetary and solar phenomena during cycle 23. Geophys. Res. Lett., 32, 104, doi: 10.1029/2004GL021691. [Link]

Richardson, I. G. and H. V. Cane, 2010: Near-earth interplanetary coronal mass ejections during solar cycle 23 (1996-2009): Catalog and summary of properties. Sol. Phys., 264, 189-237, doi: 10.1007/s11207-010-9568-6. [Link]

Riley, P., C. Schatzman, H. V. Cane, I. G. Richardson, and N. Gopalswamy, 2006: On the rates of coronal mass ejections: Remote solar and in situ observations. Astrophys. J., 647, 648-653, doi: 10.1086/505383. [Link]

Tsurutani, B. T. and W. D. Gonzalez, 1997: The interplanetary causes of magnetic storms: A review. In: Tsurutani, B. T., W. D. Gonzalez, and Y. Kamide (Eds.), Magnetic Storms, Geophys. Monogr. Ser., 98, 77-89, doi: 10.1029/GM098p0077. [Link]

Wang, Y., C. Chen, B. Gui, C. Shen, P. Ye, and S. Wang, 2011: Statistical study of coronal mass ejection source locations: Understanding CMEs viewed in coronagraphs. J. Geophys. Res., 116, 104, doi: 10.1029/2010 JA016101. [Link]

Webb, D. F., E. W. Cliver, N. U. Crooker, O. C. St. Cyr, and B. J. Thompson, 2000: Relationship of halo coronal mass ejections, magnetic clouds, and magnetic storms. J. Geophys. Res., 105, 7491-7508, doi: 10.1029/1999 JA000275. [Link]

Wu, C. C. and R. P. Lepping, 2002: Effects of magnetic clouds on the occurrence of geomagnetic storms: The 
first 4 years of WIND. J. Geophys. Res., 107, 1314, SMP19-1-SMP19-8, doi: 10.1029/2001JA000161. [Link]

Wu, C. C. and R. P. Lepping, 2007: Comparison of the characteristics of magnetic clouds and magnetic cloud-like structures for the events of 1995-2003. Sol. Phys., 242, 159-165, doi: 10.1007/s11207-007-0323-6. [Link]

Wu, C. C. and R. P. Lepping, 2008: Geomagnetic activity associated with magnetic clouds, magnetic cloud-like structures and interplanetary shocks for the period 1995-2003. Adv. Space Res., 41, 335-338, doi: 10.10 16/j.asr.2007.02.027. [Link]

Wu, C. C. and R. P. Lepping, 2011: Statistical comparison of magnetic clouds with interplanetary coronal mass ejections for solar cycle 23. Sol. Phys., 269, 141-153, doi: 10.1007/s11207-010-9684-3. [Link]

Wu, C. C., R. P. Lepping, and N. Gopalswamy, 2003: Variations of magnetic clouds and CMEs with solar activity cycle. In: Wilson A. (Ed.), Proceedings of Solar Variability as an Input to the Earth's Environment, International Solar Cycle Studies (ISCS) Symposium, 23 - 28 June 2003, Tatransk Lomnica, Slovak Republic, ESA SP-535, Noordwijk: ESA Publications Division, ISBN 92-9092-845-X, 2003, 429-432.

Wu, C. C., R. P. Lepping, and N. Gopalswamy, 2006: Relationships among magnetic clouds, CMEs, and geomagnetic storms. Sol. Phys., 239, 449-460, doi: 10.1007/ s11207-006-0037-1. [Link]

Wu, C. C., C. D. Fry, M. Dryer, S. T. Wu, B. Thompson, K. Liou, and X. S. Feng, 2007a: Three-dimensional global simulation of multiple ICMEs' interaction and propagation from the Sun to the heliosphere following the 25-28 October 2003 solar events. Adv. Space Res., 40, 1827-1834, doi: 10.1016/j.asr.2007.06.025. [Link]
Wu, C. C., C. Fry, S. T. Wu, M. Dryer, and K. Liou, 2007b: Three-dimensional global simulation of interplanetary coronal mass ejection propagation from the Sun to the heliosphere: solar event of 12 May 1997. J. Geophys. Res., 112, 104, doi: 10.1029/2006JA012211. [Link]

Yashiro, S., N. Gopalswamy, S. Akiyama, G. Michalek, and R. A. Howard, 2005: Visibility of coronal mass ejections as a function of flare location and intensity. J. Geophys. Res., 110, doi: 10.1029/2005JA011151. [Link]

Yermolaev, Y. I. and M. Y. Yermolaev, 2008: Comment on "Interplanetary origin of intense geomagnetic storms (Dst <-100 nT) during solar cycle 23" by W. D. Gonzalez et al. Geophys. Res. Lett., 35, 101, doi: 10.10 29/2007GL030281. [Link]

Yermolaev, Y. I., N. S. Nikolaeva, I. G. Lodkina, and M. Y. Yermolaev, 2010: Large-scale solar wind structures: Occurrence rate and geoeffectiveness. In: Maksimovic, M., K. Issautier, N. Meyer-Vernet, M. Moncuquet, and F. Pantellini (Eds.), Twelfth Solar Wind Conference, 21-26 June 2009, Saint-Malo, France, 1216, 648-651, doi: 10.1063/1.3395949. [Link]

Zhang, J., K. P. Dere, R. A. Howard, and V. Bothmer, 2003: Identification of solar sources of major geomagnetic storms between 1996 and 2000. Astrophys. J., 582, 520, doi: 10.1086/344611. [Link]

Zhang, J., I. G. Richardson, D. F. Webb, N. Gopalswamy, E. Huttunen, J. C. Kasper, N. V. Nitta, W. Poomvises, B. J. Thompson, C. C. Wu, S. Yashiro, and A. N. Zhukov, 2007: Solar and interplanetary sources of major geomagnetic storms (Dst $\leq-100 \mathrm{nT}$ ) during 19962005. J. Geophys. Res., 112, A10, 102, doi: 10.1029/ 2007JA012321. [Link] 